\title{
Regulating Hazardous Materials Transportation by Dual Toll Pricing
}

\author{
Tolou Esfandeh*a, Changhyun Kwon ${ }^{\dagger \mathrm{b}}$, and Rajan Batta ${ }^{\ddagger c}$ \\ ${ }^{a}$ Optym, Gainesville, FL, USA \\ ${ }^{\mathrm{b}}$ Department of Industrial and Management Systems Engineering, University of South Florida, Tampa, FL, USA \\ ${ }^{c}$ Department of Industrial and Systems Engineering, University at Buffalo, SUNY, Buffalo, NY, USA
}

October 27,2015

\begin{abstract}
We investigate dual-toll setting as a policy tool to mitigate the risk of hazardous material (hazmat) shipment in road networks. We formulate the dual-toll problem as a bi-level program wherein the upper level aims at minimizing the risk, and the lower level explores the user equilibrium decision of the regular vehicles and hazmat carriers given the toll. When the upper level objective is to minimize the risk and all links are tollable, we decompose the formulation into first-stage and second-stage, and suggest a computational method to solve each stage. Our two-stage solution methodology guarantees nonnegative valid dual tolls regardless of the solution accuracy of the first-stage problem. We also consider a general dual-toll setting problem where the regulator rather wishes to minimize a combination of risk and the paid tolls and/or some links are untollable. To solve this truly bilevel problem, we provide heuristic algorithms that decompose the problem into subproblems each being solved by a line search. Case studies based on the Sioux Falls network illustrate the insights on the dual-toll policies.
\end{abstract}

Keywords: hazardous material transportation; toll setting; non-convex optimization; bi-level programming

\section{Introduction}

Despite the extensive use of hazardous material (hazmat) from fueling our vehicles and heating our homes to farming, medical and manufacturing purposes, they are potentially disastrous to the people and the environment. Common examples are explosives, gases, flammable liquids and poisonous substances shipped by trucks, trains, vessels, and planes containing undesirable consequences in the event that they release or explode due to an accident. According to the U.S. Department

*tolou.esfandeh@optym.com

${ }^{\dagger}$ chkwon@usf .edu; Corresponding author

${ }^{\ddagger}$ batta@buffalo.edu 
of Transportation Pipeline and Hazardous Materials Agency, among the close to 1 million daily shipments of hazmats crisscrossing the United States, during the year 2013, 16,769 hazmat incidents have been recorded. Nevertheless, these incidents caused a total of 12 fatalities, 23 major and 123 minor injuries and damages of over $\$ 114$ million. These incidents underscore the importance of overseeing safe, reliable and environmentally sound hazmat transportation. This study specifically focuses on regulating hazmat transportation in road networks motivated by the fact that in $85 \%$ of the hazmat incidents truck has been used as the mode of transportation.

To mitigate hazmat transport risk, authorities usually attempt to separate hazmat flow from regular traffic flow while directing hazmat trucks to less populated areas. Two groups of policies are available to the governments: network design and toll setting. In the former approach, the authority restricts hazmat carriers from using certain road segments by imposing (permanent or time-based) curfews (Kara and Verter, 2004; Erkut and Alp, 2007; Erkut and Gzara, 2008; Bianco et al., 2009). The latter approach diverts hazmat carriers to less risky areas based on their economical and time preferences by assigning tolls to road segments (Marcotte et al., 2009; Wang et al., 2012; Bianco et al., 2015). While toll pricing is most often used to reduce traffic congestion (Arnott and Small, 1994; Hearn and Ramana, 1998; Johansson-Stenman and Sterner, 1998), toll setting (TS) policy also provides a flexible and effective hazmat transport risk mitigation tool (Marcotte et al., 2009).

The majority of existing literature on hazmat risk mitigation policies focuses either on the restrictive network design policy, or single toll pricing that excludes the inevitable impact of regular vehicles on hazmat risk. Even though Wang et al. (2012) proposed a dual toll pricing problem to regulate both types of traffic, their model assumes a linear travel delay function for analytical tractability, which does not capture the regular traffic congestion properly. Our dual-toll pricing model uses a nonlinear travel delay function used by the U.S. Bureau of Public Roads.

We develop computational methods for both first-best and second-best dual-toll pricing policies. When every road link is tollable, it is called first-best, and when some links are not tollable, it is second-best. For the first-best toll pricing, we extend the two-stage approaches of Yang and Huang (2004) and Marcotte et al. (2009) that are based on inverse optimization. As the first-best toll pricing policy seeks a nonnegative toll vector that can lead traffic flow to the desired optimal traffic pattern, it naturally involves the notion of inverse optimization (Ahuja and Orlin, 2001). In the conventional inverse optimization approaches for toll-pricing, we must obtain an optimal solution to the first-stage problem to ensure the existence of a valid toll. In our work we consider nonlinearity of the travel delay function, which makes the objective function of the first stage problem non-convex; hence an optimal solution is difficult to find. Nevertheless, we propose a solution method to guarantee the existence of a valid toll even with an approximate solution to the first stage problem. When only a subset of links is tollable, we formulate the second-best toll pricing problem as a mathematical program with equilibrium constraints (MPEC), and propose a two-step equilibrium-decomposition-optimization (2-step EDO) method that extends the work of Suwansirikul et al. (1987).

In the literature of hazmat routing, risk measurement typically relies on two link attributes: 
accident probability and accident consequence. The accident consequence can be the population, which may be measured using the $\lambda$-neighborhood concept (Batta and Chiu, 1988), or it can also include damages to individuals who are directly involved in an incident as well as damage to the environment and properties (Abkowitz and Cheng, 1988). Various measures of hazmat accident risk can be defined by considering different distributions for these two link attributes such as quantilebased measures (Kang et al., 2011; Toumazis et al., 2013). We use a duration-population-frequency risk measure to model the dependence of hazmat risk on congestion induced by regular traffic.

The rest of this paper is organized as follows. Section 2 reviews technical contributions related to our research. Section 3 develops the dual toll pricing model. Section 4 is devoted to our solution methodology for the first-best dual-toll pricing. Section 5 explains the 2 -step EDO method to solve the second-best dual-toll pricing. We present an application on the realistic road network of Sioux Falls in Section 6. Finally, Section 7 concludes the paper and suggests some future research directions.

\section{Review of Related Technical Contributions}

We review technical contributions in past research efforts for both regular and hazmat traffic control that use toll pricing. We build upon these techniques in developing our solution methodology.

\subsection{Regular Traffic Control}

In regular traffic control, the primary objective of the network administrator is to minimize a certain system total cost function such as the total travel time or the total emissions-denoted by system optimum. Consider a directed network $\mathbb{G}(\mathcal{N}, \mathcal{A})$, with a set of nodes $\mathcal{N}$, and a set of $\operatorname{arcs} \mathcal{A}$. Let $f_{i j}\left(v_{i j}\right)$, a function of arc traffic volume $v_{i j}$, denote such a performance measure in each arc $(i, j) \in \mathcal{N}$ that the network administrator wants to minimize. We assume there is travel demand $d^{w}$ for each O-D pair $w$ in the set of O-D pairs $\mathcal{W}$. The administrator's problem is written as follows:

$$
\min _{v \in V} \sum_{(i, j) \in \mathcal{A}} f_{i j}\left(v_{i j}\right)
$$

where

$$
\begin{aligned}
& V:=\left\{v: v_{i j}=\sum_{w \in \mathcal{W}} y_{i j}^{w} \quad \forall(i, j) \in \mathcal{A},\right. \sum_{j:(i, j) \in \mathcal{A}} y_{i j}^{w}-\sum_{j:(j, i) \in \mathcal{A}} y_{j i}^{w}=b_{i}^{w}, \quad \forall i \in \mathcal{N}, \forall w \in \mathcal{W}, \\
&\left.y_{i j}^{w} \geq 0, \quad \forall(i, j) \in \mathcal{A}, \forall w \in \mathcal{W}\right\},
\end{aligned}
$$


$y_{i j}^{w}$ is the traffic flow in arc $(i, j)$ for O-D pair $w, b_{i}^{w}=d^{w}$ if node $i$ is the origin of O-D pair $w$, $b_{i}^{w}=-d^{w}$ if node $i$ is the destination of O-D pair $w$, and $b_{i}^{w}=0$ for all other intermediate nodes. We let an optimal solution to the administrator's problem (1) to be $\bar{v}$.

While $\bar{v}$ is the desired traffic flow of the administrator, network users will not necessarily follow the desire. Instead, they are often interested in their own benefits; hence a game-theoretic model is necessary. Given a tolling scheme from the administrator, according to the Wardrop's first principle (e.g. Florian and Hearn, 1995), the users, in an attempt to minimize their individual travel cost, will take the User Equilibrium flow pattern. When arc traveling time/cost is $c_{i j}\left(v_{i j}\right)$, the user equilibrium of network users can be modeled as a variational inequality (e.g. Dafermos, 1980). Let us introduce toll prices $\tau_{i j}$ for each $\operatorname{arc}(i, j) \in \mathcal{A}$. Therefore, the objective of the toll pricing problem is to make the desired flow pattern $\bar{v}$ the tolled-user equilibrium flow that satisfies the following variational inequality:

$$
\sum_{(i, j) \in \mathcal{A}}\left(c_{i j}\left(\bar{v}_{i j}\right)+\tau_{i j}\right)\left(v_{i j}-\bar{v}_{i j}\right) \geq 0, \quad \forall v \in V .
$$

Any toll vector $\tau$ that makes (3) hold is called a valid toll (Hearn and Ramana, 1998). Bergendorff et al. (1997) develop and prove the following property:

Lemma 1. For $\tau$ to be a valid toll vector for $\bar{v} \in V$, there should exist a vector $\lambda$ such that

$$
\begin{array}{r}
c_{i j}\left(\bar{v}_{i j}\right)+\tau_{i j} \geq \lambda_{i}^{w}-\lambda_{j}^{w}, \quad \forall(i, j) \in \mathcal{A}, w \in \mathcal{W}, \\
\sum_{(i, j) \in \mathcal{A}}\left[c_{i j}\left(\bar{v}_{i j}\right)+\tau_{i j}\right] \bar{v}_{i j}=\sum_{i \in \mathcal{N}} \sum_{w \in \mathcal{W}} \lambda_{i}^{w} b_{i}^{w} .
\end{array}
$$

We notice that to obtain constraints (4) and (5), one can construct the Lagrangian of variational inequality (3), and derive the KKT conditions.

Usually, there are multiple valid tolls and one may choose a toll based on another criteria. A popular choice is to minimize the total toll collected. Hence, for a given $\bar{v}$, we solve:

$$
\operatorname{RTP}(\bar{v}): \quad \min _{\tau \geq 0} \sum_{(i, j) \in \mathcal{A}} \bar{v}_{i j} \tau_{i j}
$$

subject to (4) and (5).

The regular-toll problem (RTP) is an instance of inverse optimization (IO) problems (e.g. Ahuja and Orlin, 2001) to find toll prices. Mathematically, arc tolls can be positive or negative (subsidy). However, it is impractical to consider subsidies when trying to regulate traffic. The existence of a nonnegative valid toll vector $\tau \geq 0$ is assured by the following theorem due to Yin and Lawphongpanich (2006):

Theorem 1. If $f_{i j}(\cdot)$ is a monotically increasing function for all $(i, j) \in \mathcal{A}$, there exists a nonnegative valid toll vector $\tau \geq 0$ for the traffic pattern $\bar{v}$. Therefore, problem $\operatorname{RTP}(\bar{v})$ has a feasible solution.

A key to Theorem 1 is that $\bar{v}$ is an optimal solution to (1). 


\subsection{Hazmat Traffic Regulation}

To guide hazmat traffic to a certain desired pattern, we can follow an analogous process as introduced by Marcotte et al. (2009). On the directed network $\mathbb{G}(\mathcal{N}, \mathcal{A})$, we let $\mathcal{S}$ denote the set of shipments (similar to the set of O-D pairs for regular traffic case), $n^{s}$ the number of trucks for each shipment $s \in \mathcal{S}$, and $x_{i j}^{s}$ a binary routing variable corresponding to shipment $s$ on $\operatorname{arc}(i, j) \in \mathcal{A}$. For a certain performance measure $g_{i j}(\cdot)$ for each arc $(i, j) \in \mathcal{A}$, the hazmat network administrator's problem can be written as follows:

$$
\min _{x \in X} \sum_{s \in \mathcal{S}} \sum_{(i, j) \in \mathcal{A}} g_{i j}\left(x_{i j}^{s}\right)
$$

where

$$
X:=\left\{x: \sum_{j:(i, j) \in \mathcal{A}} x_{i j}^{s}-\sum_{j:(j, i) \in \mathcal{A}} x_{j i}^{s}=e_{i}^{s} \quad \forall i \in \mathcal{N}, s \in \mathcal{S}, \quad x_{i j}^{s} \in\{0,1\} \quad \forall(i, j) \in \mathcal{A}, s \in \mathcal{S}\right\},
$$

$e_{i}^{s}=1$ if node $i$ is the origin of shipment $s, e_{i}^{s}=-1$ if node $i$ is the destination of shipment $s$, and $e_{i}^{s}=0$ for all other intermediate nodes $i$. We let $\bar{x}$ denote an optimal solution to problem (7).

As opposed to the user equilibrium in the regular traffic case wherein an O-D pair flow can split in many routes, we assume hazmat carriers use the shortest path for all trucks of each shipment. Thus, with $c_{i j}$ a nonnegative travel time for each $\operatorname{arc}(i, j) \in \mathcal{A}$, and a hazmat toll vector $t$, hazmat carriers determine their routes by solving the following multi-commodity shortest path problem:

$$
\min _{x \in X} \sum_{s \in \mathcal{S}} \sum_{(i, j) \in \mathcal{A}} n^{s}\left(c_{i j}+t_{i j}\right) x_{i j}^{s}
$$

A valid hazmat toll vector $t$ makes the desired hazmat traffic pattern $\bar{x}$ an optimal solution to (9). Referring to Marcotte et al. (2009), Section 4, we can characterize a valid hazmat toll:

Lemma 2. For $t$ to be a valid toll vector for $\bar{x} \in X$, there should exist a vector $\pi$ such that

$$
\begin{aligned}
\pi_{i}^{s}-\pi_{j}^{s} \leq n^{s}\left(c_{i j}+t_{i j}\right) & \forall(i, j) \in \mathcal{A}, s \in \mathcal{S}, \\
\bar{x}_{i j}^{s}\left(\pi_{i}^{s}-\pi_{j}^{s}-n^{s}\left(c_{i j}+t_{i j}\right)\right)=0 & \forall(i, j) \in \mathcal{A}, s \in \mathcal{S} .
\end{aligned}
$$

Similar to the regular traffic case, multiple valid toll vectors may exist and one can choose a toll to minimize the total toll collected:

$$
\begin{aligned}
\operatorname{HTP}(\bar{x}): & \min _{t \geq 0} \sum_{s \in \mathcal{S}} \sum_{(i, j) \in \mathcal{A}} n^{s} \bar{x}_{i j}^{s} t_{i j}, \\
& \text { subject to }(10) \text { and (11). }
\end{aligned}
$$

The following theorem guarantees the existence of a nonnegative valid toll vector $t \geq 0$ : 


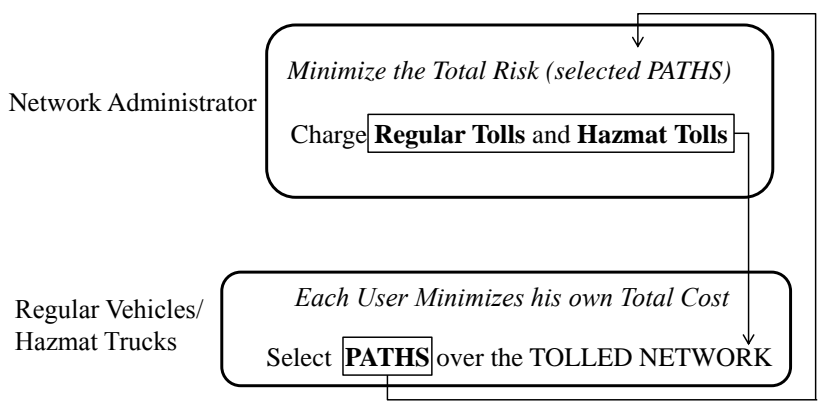

Figure 1: Hazardous-Network Dual Toll Pricing Problem

Theorem 2. If $g_{i j}(\cdot)$ is a monotonically increasing function for all $(i, j) \in \mathcal{A}$, there exists a nonnegative valid toll vector $t \geq 0$ for the traffic pattern $\bar{x}$.

Proof. We can revise the proof of Marcotte et al. (2009, Proposition 1). Although the original proof is written for a linear program with nonnegative cost coefficients, the same argument applies to any increasing function $g_{i j}(\cdot)$.

Again, a key to Theorem 2 is that $\bar{x}$ is an optimal solution to (7).

\section{Hazardous-Network Dual Toll Pricing Problem}

We introduce the dual-toll pricing problem to minimize the risk of hazmat transportation. In our network, there are two distinct groups of decision makers: the local government, and the network users including regular vehicles' drivers and hazmat carriers. To capture this leader-follower relationship, we use a bilevel framework to represent our model with the government in level 1 and the users in level 2 as depicted in Figure 1.

Once the government decides on the toll charges in level 1, two different sets of users (i.e., regular traffic and hazmat carriers) will minimize their total travel cost in level 2. Specifically, the regular traffic will take the tolled user equilibrium flow, and apart from them, the hazmat carriers will choose the shortest paths between every origin and destination pair considering both time and economical preferences. In response to such behavior, the government can indirectly influence the users' decisions through toll pricing. We seek a dual toll policy that produces a mutually acceptable solution.

\subsection{A Bilevel Model}

We represent the existing road system by the directed network $\mathbb{G}(\mathcal{N}, \mathcal{A})$, with a set of nodes $\mathcal{N}$, and a set of $\operatorname{arcs} \mathcal{A}$. Consider a regular traffic demand $d^{w}$ for each O-D pair $w$ in regular O-D pair set $\mathcal{W}$. In addition, there is a set $\mathcal{S}$ of O-D shipments for hazmat traffic with each shipment $s \in \mathcal{S}$ representing a specific hazmat type with a certain level of risk exposure. Let $n^{s}$ be the number of trucks needed to complete every shipment $s \in \mathcal{S}$. Finally, let $\rho_{i j}$ denote a measure of hazmat 
Table 1: Mathematical notation

\begin{tabular}{cl}
\hline Notation & Description \\
\hline $\mathcal{A}$ & Set of arcs \\
$\mathcal{N}$ & Set of nodes \\
$\mathcal{W}$ & Set of O-D pairs for regular vehicles \\
$d^{w}$ & Demand of regular vehicles for O-D pair $\mathcal{W}$ \\
$y_{i j}^{w}$ & Regular traffic flow variable in arc $(i, j)$ for O-D pair $w$ \\
$v_{i j}$ & Total regular traffic volume in arc $(i, j)$ \\
$b_{i}^{w}$ & Equals $d^{w},-d^{w}$, or 0 depending if node $i \in \mathcal{N}$ is the origin, \\
& the destination or an intermediate node for O-D pair $w$ \\
$\mathcal{S}$ & Set of hazmat O-D shipments \\
$n^{s}$ & Number of trucks for shipment $s \in \mathcal{S}$ \\
$x_{i j}^{s}$ & Binary variable taking the value 1 if arc $(i, j) \in \mathcal{A}$ is used for shipment $s \in \mathcal{S}$ \\
$e_{i}^{s}$ & Equals $1,-1$, or 0 depending if node $i \in \mathcal{N}$ is the origin, \\
& the destination or an intermediate node for shipment $s$ \\
$\rho_{i j}$ & Number of people exposed on arc $(i, j)$ when a hazmat accident occurs \\
$c_{i j}$ & A nonnegative arc travel time for each arc $(i, j) \in \mathcal{A}$ \\
$\tau_{i j}$ & Continuous variable representing the toll charged to regular vehicles on arc $(i, j) \in \mathcal{A}$ \\
$t_{i j}$ & Continuous variable representing the toll charged to hazmat trucks on arc $(i, j) \in \mathcal{A}$ \\
$\sigma_{R}$ & Parameter converting toll unit into travel time unit perceived by Regular vehicles \\
$\sigma_{H}$ & Parameter converting toll unit into travel time unit perceived by Hazmat trucks \\
\hline
\end{tabular}

transportation risk in $\operatorname{arc}(i, j) \in \mathcal{A}$, for example, population along each arc. Table 1 provides a summary of the notation used in the formulations.

We make two simplifying assumptions for analytical tractability of our model. First, we assume that network users have perfect information of the current status of the network. Second, there is no stochasticity in the travel time and the behavior of users implying that our model is deterministic.

By imposing separate toll policies $\tau$ and $t$, we guide regular and hazmat traffic to a solution of network administrator's problem of the following form:

$$
\min _{v \in V, x \in X} z(v, x)=\sum_{s \in \mathcal{S}} \sum_{(i, j) \in \mathcal{A}} n^{s} c_{i j}\left(v_{i j}\right) \rho_{i j} x_{i j}^{s}
$$

with $V$ and $X$ being as constraint sets (2) and (8), respectively. The objective function $z(v, x)$ is called a duration-population-frequency measure of hazmat exposure in arc $(i, j)$ (Wang et al., 2012) as it involves the product of $c_{i j}, \rho_{i j}$ and $n^{s}$. To determine the travel time on each link given the flow, we use the US Bureau of Public Roads (BPR) function. Algebraically, the function is of the form $c_{i j}\left(v_{i j}\right)=c_{i j}^{0}\left(1+0.15\left(v_{i j} / l_{i j}\right)^{4}\right)$, where $v_{i j}$ is the amount of the flow on link $(i, j)$. In a road network, the number of hazmat trucks is relatively small when compared to the flow of regular traffic. Therefore, in calculating the link travel time, congestion induced by the traffic flow of hazmat trucks can be ignored. Consequently, we assume the link travel delay function $c_{i j}$ to be only a function of regular traffic flow $v_{i j}$. The remaining parameters, $c_{i j}^{0}$ and $l_{i j}$, represent the 
free-flow travel time and capacity for link $(i, j)$, respectively.

Once the administrator makes a decision on toll charges for regular and hazmat traffic (upper level), the regular vehicles' drivers follow the tolled-user equilibrium flow pattern that satisfies the variational inequality of the form (3), and hazmat carriers take the shortest path between each specified O-D pair by solving equation (9).

The hazardous-network dual toll pricing problem lends itself to a bilevel programming formulation of the following form:

$$
\text { (TS) } \min _{v \in V, x \in X, \tau \geq 0, t \geq 0} z(v, x)=\sum_{s \in \mathcal{S}} \sum_{(i, j) \in \mathcal{A}} n^{s} c_{i j}\left(v_{i j}\right) \rho_{i j} x_{i j}^{s}
$$

where $v_{i j}$ solves the following tolled-user equilibrium problem:

$$
\text { (R) } \quad \sum_{(i, j) \in \mathcal{A}}\left(c_{i j}\left(v_{i j}\right)+\sigma_{R} \tau_{i j}\right)\left(v_{i j}^{\prime}-v_{i j}\right) \geq 0, \quad \forall v^{\prime} \in V
$$

with $V$ being as set (2), and simultaneously $x_{i j}^{s}$ solves the shortest path problem:

$$
\text { (H) } \min _{x \in X} \sum_{s \in \mathcal{S}} \sum_{(i, j) \in \mathcal{A}} n^{s}\left(c_{i j}\left(v_{i j}\right)+\sigma_{H} t_{i j}\right) x_{i j}^{s}
$$

with $X$ being as set (8). Overall, equations (14)-(16) represent the bilevel model denoted by problem (TS). We note that problem $(\mathrm{H})$ contains the decision variables of the regular vehicles' problem $(\mathrm{R})$, i.e., $v_{i j}$, which implies that hazmat carriers' route choice is influenced by regular traffic flow through the link travel time function $c_{i j}\left(v_{i j}\right)$. When some links are untollable as in the second-best dual-toll pricing problem, corresponding toll variables, $\tau_{i j}$ and $t_{i j}$, will be forced to be zero.

Problem (TS) is a mathematical program with equilibrium constraints (MPEC), which is in general very difficult to solve. Section 4 develops an efficient two-stage method for the first-best dual-toll pricing problem. For the second-best dual-toll pricing problem, Section 5 develops a heuristic algorithm.

\section{Two-Stage Inverse-Optimization Approach}

We exploit specific structural properties to demonstrate that problem (TS) as an MPEC is equivalent to a two-stage problem which can be solved efficiently, using Theorems 1 and 2 .

The first stage problem corresponds to determining the minimum-risk flow pattern, i.e., the administrator's ideal solution. The minimum-risk problem (MR) is comprised of the objective function of problem (TS), i.e., equation (14), and the flow conservation constraints (2) and (8), without considering toll vectors. That is, we solve:

$$
\text { (MR) } \min _{v \in V, x \in X} z(v, x)=\sum_{s \in \mathcal{S}} \sum_{(i, j) \in \mathcal{A}} n^{s} c_{i j}\left(v_{i j}\right) \rho_{i j} x_{i j}^{s} .
$$


for which we can relax the binarity conditions on $x$. Note that (MR) is a non-convex problem. We can prove, by a counter-example, that problem (17) is not even pseudo-convex or quasi-convex. Thus, finding an optimal solution to (MR) is challenging.

An important issue surrounding the existence of nonnegative valid toll vector pairs, i.e., $\tau$ and $t$, is that our numerical solution to (17) may be sub-optimal. Since Theorems 1 and 2 require optimal solutions, the existence of a valid toll is not guaranteed for an approximate numerical solution to (MR). To overcome this issue, we propose a simple post-iteration to the numerical solution to (MR).

We let $\breve{v} \in V$ and $\breve{x} \in X$ denote an approximate solution to (17) obtained using an algorithm. Then the post-iteration is that we solve two following optimization problems:

$$
\begin{aligned}
& \breve{v}^{l+1}=\arg \min _{v \in V} z\left(v, \breve{x}^{l}\right) \\
& \breve{x}^{l+1}=\arg \min _{x \in X} z\left(\breve{v}^{l+1}, x\right),
\end{aligned}
$$

until convergence, starting with an initial solution $\breve{x}^{0}=\breve{x}$ when $l=0$. Note that (18) is an instance of convex traffic assignment problem which can be solved efficiently using, e.g., the Frank-Wolfe algorithm (Frank and Wolfe, 1956) and its variants, and (19) is a pure network flow problem (one shortest-path problem per carrier). Finally, let $(\tilde{v}, \tilde{x})$ represent a solution to the post-iteration phase.

Despite the simplicity, the post-iteration has two major outcomes. First, although the pair $(\tilde{v}, \tilde{x})$ may not be an optimal solution to (17), the risk value corresponding to $(\tilde{v}, \tilde{x})$ is at least as good as the one induced by $(\breve{v}, \breve{x})$. Second, after the post-iteration (18) and (19), we obtain the following existence results:

Theorem 3. For any approximate solution $\breve{v} \in V$ and $\breve{x} \in X$ to (MR), there exists a nonnegative valid dual-toll pair $(\tau, t) \geq 0$ for the traffic pattern $(\tilde{v}, \tilde{x})$ as obtained by (18) and (19).

Proof. Since $z(\cdot, \breve{x})$ is an increasing function, there exists a nonnegative valid regular toll $\tau$ by Theorem 1. Similarly the existence of a nonnegative valid hazmat toll $t$ is assured by the monotonicity of $z(\tilde{v}, \cdot)$ and Theorem 2 .

After obtaining $\tilde{v}$ and $\tilde{x}$, in the second stage we use the inverse optimization problems $\operatorname{RTP}(\tilde{v})$ as equation (6), and $\operatorname{HTP}(\tilde{x})$ as equation (12) to determine toll vectors $\tau$ and $t$, whose existence is guaranteed by Theorem 3. We note that the inverse optimization enforces achievement of dual-toll vector $(\tau, t)$ such that $\tilde{v}$ and $\tilde{x}$ are a tolled-user equilibrium and shortest-paths, respectively. The flowchart of the entire two-stage process is illustrated in Figure 2 for easy reference. Therefore, the original problem (TS) is analytically equivalent to this two-stage problem. In what follows, we focus on the computational methods to separately solve each stage of the two-stage problem.

\subsection{The First Stage Problem}

Note that (MR) is a non-convex optimization problem with two disjoint linear constraint sets $V$ and $X$. We further observe that for any given $x, \min _{v \in V} z(v, x)$ is an easy convex minimization with 


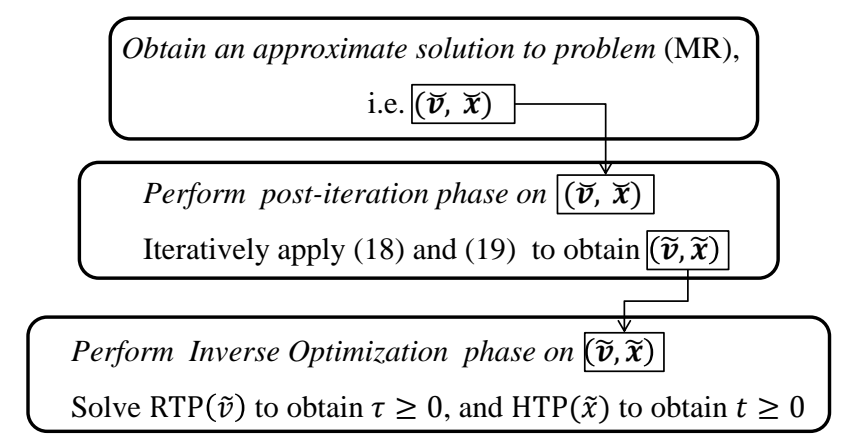

Figure 2: The Two-Stage Process Flowchart for Problem (TS)

linear constraints, and for any given $v, \min _{x \in X} z(v, x)$ is the shortest path problem which can be easily solved. However, a cyclic minimization approach will not guarantee optimality. On the other hand, other methods such as branch-and-bound which guarantee the optimality are likely to be computationally expensive.

For solving the first stage problem, we use the Modified Frank-Wolfe algorithm proposed by Fukushima (1984), which is devised for convex optimization problems. Although there is no guarantee on the optimality for the non-convex problem (MR), it is computationally efficient. We will show that an approximate solution is enough for the dual-toll pricing purpose, and there always exists a nonnegative dual-toll pair if a simple post-iteration is applied at the end of the Frank-Wolfe iterations. The details of the Modified Frank-Wolfe algorithm applied in problem (MR) are described in a supplementary document (Appendix A, Part I).

The Modified Frank-Wolfe algorithm is a heuristic approach for the non-convex problem (MR) and does not guarantee the global optimality. To study the quality of the solution provided by Modified Frank-Wolfe algorithm, we use two linearization approaches whose solution possesses the desirable property of global optimality. We then use those solutions to justify our results from the heuristic method. Note that throughout Section 4.1, we use the terms 'Modified Frank-Wolfe' and 'heuristic' interchangeably.

The linearization idea is rooted in the work of Wang and Lo (2010) who transform the network design problem with equilibrium constraints into a mixed-integer linear program that may be solved optimally by an optimization solver. We incorporate a similar logic to our model to approximate the travel time function $c_{i j}\left(v_{i j}\right)$ with a set of piecewise-linear functions.

In the first linearization approach, referred to as piecewise-linear model 1 , transformation of the nonlinear objective function (17) involves two parts. The first is to linearize the travel time function and the second is to linearize the resultant bilinear terms. For doing so, we partition the feasible domain of $v_{i j}$ into $N$ segments each denoted as $[n]$. We note that the larger the number of partitions, the finer the discretization adopted, resulting in a more accurate global optimal solution. Then we modify the original idea of Wang and Lo (2010) for the single variable function $c_{i j}\left(v_{i j}\right)$, and specify a linear function to approximate $c_{i j}\left(v_{i j}\right)$ within each region $[n]$. However, after replacing the linear transformation of the travel time in the objective function (17), the result is still nonlinear due 
Table 2: Test networks

\begin{tabular}{rrrrr}
\hline Test Problem & No. of & No. of & \multicolumn{2}{c}{ No. of OD pairs } \\
\cline { 4 - 5 } & Nodes & Links & Regular & Hazmat \\
\hline 8-node network & 8 & 13 & 27 & 2 \\
10-node network & 10 & 20 & 39 & 3 \\
15-node network & 15 & 28 & 97 & 4 \\
\hline
\end{tabular}

to the bilinear terms. Every bilinear term is denoted by the product of a nonnegative continuous and a binary variable, namely $v_{i j}$ and $x_{i j}^{s}$. Therefore, we adapt the approach proposed by Gupte et al. (2013) to replace the bilinear term with an equivalent continuous variable. Eventually, by combining all the constraints required for transformations, we can convert nonlinear problem (MR) to a mixed-integer linear problem referred to as (MILP-1).

The second linearization approach in transformation of the objective function (17) to a linear problem considers the entire nonlinear term in problem (MR) as a single function with two variables, namely $v_{i j}$ and $x_{i j}^{s}$. Therefore, the linearization relies solely on the piecewise technique introduced by Wang and Lo (2010). In essence, in piecewise-linear model 2, we incorporate their idea of linearizing a two-variable nonlinear function while taking advantage of the binary nature of some variables. Consequently, another equivalent MILP model is obtained for problem (MR) which we refer to as (MILP-2). The comprehensive descriptions of the two linearization approaches are delegated to the supplementary document (Appendix A, Parts II and III).

To compare the Modified Frank-Wolfe algorithm with MILP-1 and MILP-2, we consider three networks for our test problem generation, having 8, 10, and 15 nodes, respectively. Table 2 provides a summary of these networks, with additional details in the supplementary document (Appendix C). We used Java as the programming language. When required, for solving LP or MILP, we use CPLEX 12.5.1 integrated with Java platform. All experiments were performed on an Intel(R) Processor 2.99 GHz computer. The supplementary document (Appendix A, Part IV) entails the complete version of our extensive experiments on the efficiency of the heuristic algorithm and the two exact models. Here, we underscore our most significant observations as well as the final comparative analysis.

To verify the solution quality of the heuristic we present a summary of our computational results for the three algorithms, namely Modified Frank-Wolfe, Piecewise Linear Model 1 and 2. We compare the quality of the solutions provided by each of the algorithms as well as the time required to obtain such solutions. Notice that here by solution we denote the MR objective value found by each method which for the two piecewise-linear cases it is determined by calculating equation (17) for the flow solutions obtained by (MILP-1/2) to remove the linearization error. Specifically, for the Frank-Wolfe algorithm, starting from a few initial solutions, we choose the least Min-Risk objective value and the corresponding total computational time. Similarly, for piecewise-linear models 1 and 2, the best found MR objective value is adopted for comparison purposes. As the required computation time, we report the instant of observing the solution by MILPs. We also present the percentage gap between the solution of the heuristic with the one provided by either of the two linearization 
Table 3: Solution performance of the Modified Frank-Wolfe vs. MILP-1 and MILP-2. Run times are in seconds.

\begin{tabular}{|c|c|c|c|c|c|c|c|c|}
\hline \multirow{3}{*}{$\begin{array}{c}\text { Test } \\
\text { Problem }\end{array}$} & \multicolumn{2}{|c|}{ Modified Frank-Wolfe } & \multicolumn{2}{|c|}{ MILP-1 ${ }^{a}$} & \multicolumn{2}{|c|}{ MILP- $2^{b}$} & \multicolumn{2}{|c|}{ Gap (\%) } \\
\hline & $\mathrm{MR}^{c}$ & Run & MR & Run & MR & Run & $\mathrm{MFW}^{d}$ & MFW \\
\hline & Obj. & Time & Obj. & Time & Obj. & Time & vs. MILP-1 & vs. MILP-2 \\
\hline 8-node & 81082.36 & 2 & 81077.19 & 256 & 81077.19 & 151 & 0.006 & 0.006 \\
\hline 10-node & $16299.75 \mathrm{E} 4$ & 4 & $16298.71 \mathrm{E} 4$ & 25920 & $16298.71 \mathrm{E} 4$ & 3237 & 0.006 & 0.006 \\
\hline 15-node & 492075.36 & 227 & 486838.86 & 3379 & 487423.20 & 3325 & 1.075 & 0.954 \\
\hline
\end{tabular}

${ }^{a}$ Denotes the mixed-integer linear problem obtained by piecewise-linear model 1 ,

${ }^{b}$ Denotes the mixed-integer linear problem obtained by piecewise-linear model 2,

${ }^{c}$ Refers to the performance of the approximate solution $(\breve{v}, \breve{x})$ obtained prior to post-iteration,

${ }^{d}$ Refers to the Modified Frank-Wolfe algorithm.

approaches. The results prior to the post-iteration step are shown in Table 3 .

From Table 3 it is observed that the computation time required to obtain the solution of problem (MR) is significantly reduced under the Modified Frank-Wolfe algorithm for all problem instances. Although the piecewise-linear models produce a better exact MR objective value in all cases, the gaps between the heuristic solution and the ones provided by linearization methods are very small. Owing to the global optimality of the solution obtained from the linearization methods, we conclude that the Modified Frank-Wolfe also converges to the optimal solution of problem (MR).

The Modified Frank-Wolfe can be applied to the first-stage problem (17) to determine a regular flow pattern $\breve{v} \in V$ and a hazmat flow pattern $\breve{x} \in X$ inducing a sufficiently low level of risk to the network. In the next section, we will further show that given such an approximate minimum-risk flow pattern, it is always guaranteed to obtain a nonnegative valid toll pair.

\subsection{The Second Stage Problem}

Let $(\breve{v}, \breve{x})$ denote an approximate minimum-risk flow obtained by solving the first-stage problem (MR). To assure the existence of a nonnegative valid dual-toll vector $(\tau, t)$, the post-iteration step is carried out. That is the optimization problems (18) and (19) are solved using the Frank-Wolfe algorithm (Frank and Wolfe, 1956), and a shortest path algorithm such as Dijkstra's, respectively, to obtain $(\tilde{v}, \tilde{x})$. Now according to Theorem 3 , we can determine a nonnegative regular toll vector $\tau$ by solving

$$
\begin{gathered}
\operatorname{RTP}(\tilde{v}): \quad \min _{\tau \geq 0} \sum_{(i, j) \in \mathcal{A}} \tilde{v}_{i j} \tau_{i j}, \\
\text { subject to } \\
c_{i j}\left(\tilde{v}_{i j}\right)+\sigma_{R} \tau_{i j} \geq \lambda_{i}^{w}-\lambda_{j}^{w}, \quad \forall(i, j) \in \mathcal{A}, w \in \mathcal{W}, \\
\\
\sum_{(i, j) \in \mathcal{A}}\left[c_{i j}\left(\tilde{v}_{i j}\right)+\sigma_{R} \tau_{i j}\right] \tilde{v}_{i j}=\sum_{i \in \mathcal{N}} \sum_{w \in \mathcal{W}} \lambda_{i}^{w} b_{i}^{w},
\end{gathered}
$$


and a nonnegative hazmat toll vector $t$ using

$$
\begin{aligned}
& \operatorname{HTP}(\tilde{x}): \quad \min _{t \geq 0} \sum_{s \in \mathcal{S}} \sum_{(i, j) \in \mathcal{A}} n^{s} \tilde{x}_{i j}^{s} t_{i j}, \\
& \quad \text { subject to } \\
& \pi_{i}^{s}-\pi_{j}^{s} \leq n^{s}\left(c_{i j}+\sigma_{H} t_{i j}\right) \quad \forall(i, j) \in \mathcal{A}, s \in \mathcal{S}, \\
& \tilde{x}_{i j}^{s}\left(\pi_{i}^{s}-\pi_{j}^{s}-n^{s}\left(c_{i j}+\sigma_{H} t_{i j}\right)\right)=0 \quad \forall(i, j) \in \mathcal{A}, s \in \mathcal{S} .
\end{aligned}
$$

Both inverse optimization problems $\operatorname{RTP}(\tilde{v})$ and $\operatorname{HTP}(\tilde{x})$ are linear programs which can be solved efficiently.

To computationally test the validity of our claim, we also implemented the second stage on the problem instances described in Section 4.1. Our numerical experiments reveal that there always exists a nonnegative valid dual-toll vector $(\tau, t)$ resulting in a tolled-user equilibrium flow pattern for regular vehicles and shortest-path flows for hazmat trucks for which the corresponding risk is sufficiently low. That is, our results are consistent with Theorem 3.

\section{Heuristic Approaches for Truly Bilevel Cases}

So far, in problem (TS) the sole objective of the administrator is to minimize the population exposure. However, when the administrator is interested in minimizing a combination of hazmat transport risk and paid tolls, or when some links are untollable as in second-best toll pricing, the dual-toll pricing problem becomes truly bilevel, and the two-stage solution methodology is no longer a feasible approach. Therefore, in this section, we assume that in problem (TS), the objective function (14) is replaced with

$$
\begin{aligned}
\min _{v \in V, x \in X, \tau \geq 0, t \geq 0} z(v, x, \tau, t)= & \sum_{s \in \mathcal{S}} \sum_{(i, j) \in \mathcal{A}} n^{s} c_{i j}\left(v_{i j}\right) \rho_{i j} x_{i j}^{s} \\
& +\varphi\left(\sum_{(i, j) \in \mathcal{A}} \tau_{i j} v_{i j}+\sum_{s \in \mathcal{S}} \sum_{(i, j) \in \mathcal{A}} n^{s} t_{i j} x_{i j}^{s}\right),
\end{aligned}
$$

where problems $(\mathrm{R})$ and $(\mathrm{H})$ are kept same as in Section 3.1, and $\varphi$ is a parameter converting users' cost into population exposure units. If some links are untollable, we can also enforce the toll variables $\tau_{i j}$ and $t_{i j}$ in the corresponding links to be zero for the second-best toll pricing. Our proposed method still works with such untollable links with the general objective function in (26). We call this general toll setting problem as (GTS), wherein the administrator's goal of charging toll is to route network users to minimize population exposure while keeping the users' cost as low as possible.

Our main purpose is to suggest a heuristic for finding an approximate solution to problem (GTS). Our algorithm is a variant of the Equilibrium-Decomposed Optimization (EDO) heuristic first introduced for the continuous equilibrium network design problem by Suwansirikul et al. (1987). 
In the following, we briefly discuss the obstacles toward solving bi-level problem (GTS) with the conventional EDO heuristic, and present a formal statement of the altered EDO, namely the 2-Step EDO heuristic, along with the results of our numerical tests.

The major difficulty in solving problem (GTS) is due to the unknown functional forms. In particular, we note that $v(\tau)$ is a UE flow pattern wherein $v_{i j}, \forall(i, j) \in \mathcal{A}$, is an implicit function of regular toll $\tau$, i.e., $v_{i j}=v_{i j}(\tau)$. Similarly, $x_{i j}^{s}=x_{i j}^{s}(t), \forall(i, j) \in \mathcal{A}, \forall s \in \mathcal{S}$, denotes the hazmat shortest path flow pattern $x$ as an implicit function of the hazmat toll $t$. The conventional EDO of Suwansirikul et al. (1987) suggests decomposition of the problem into single variable subproblems corresponding to each arc, and simultaneously minimizing the subproblems with a one-dimensional search routine. For the two decision variable case, although one may perform a dual search for both $\tau$ and $t$, we choose to limit the one-dimensional search routines to search only for $\tau$ in order to reduce the approximation error and increase the computational efficiency.

An appropriate approach for (GTS) is to decompose it into interacting subproblems with regard to regular toll, $\tau$, one for each arc considered to be tolled. Each subproblem has the following form

subproblem of each arc $(i, j)$ :

$$
\min _{\tau} z_{i j}(\tau)=\sum_{s \in \mathcal{S}} n^{s} c_{i j}\left(v_{i j}(\tau)\right) \rho_{i j} x_{i j}^{s}(t)+\varphi\left(\tau_{i j} v_{i j}(\tau)+\sum_{s \in \mathcal{S}} n^{s} t_{i j} x_{i j}^{s}(t)\right)
$$

In fact, in equation (27), assuming that $x_{i j}(t)$ and $t$ are available from another routine, we transformed the bivariate problem into univariate unconstrained nonlinear subproblems with respect to $\tau$. The interaction among the subproblems stems from the fact that the value of $v_{i j}$ depends on the vector $\tau$ for all arc tolls. Referred to as Modified EDO heuristic, our algorithm is similar to the conventional EDO in its strategy of applying simultaneous line searches along each arc, as well as solving only one user equilibrium at each iteration. The Modified EDO algorithm is as follows:

\section{The Modified EDO}

\section{Step 0. Initialization}

- For the links considered for regular toll charge, let $L^{0}=\left(\ldots, L_{i j}^{0}, \ldots\right)$ and $U^{0}=$ $\left(\ldots, U_{i j}^{0}, \ldots\right)$ represent the initial lower and upper bounds on $\tau$, respectively.

- Solve the UE problem (R), using Frank-Wolfe algorithm, once for $\tau=L^{0}$ to obtain the regular flow vector $v\left(L^{0}\right)$, and once for $\tau=U^{0}$ to obtain $v\left(U^{0}\right)$.

- Obtain hazmat shortest path flow $\left.x(t)\right|_{L^{0}}$ and hazmat toll $\left.t\right|_{L^{0}}$ corresponding to $v\left(L^{0}\right)$. Similarly, obtain $\left.x(t)\right|_{U^{0}}$ and hazmat toll $\left.t\right|_{U^{0}}$ corresponding to $v\left(U^{0}\right)$. For doing so, solve the following bi-level model for hazmat toll setting given a regular flow vector $\check{v}$ :

$$
\text { (HTS) } \min _{x \in\{0,1\}, t \geq 0} \sum_{s \in \mathcal{S}} \sum_{(i, j) \in \mathcal{A}} n^{s} c_{i j}\left(\check{v}_{i j}\right) \rho_{i j} x_{i j}^{s}+\varphi \sum_{s \in \mathcal{S}} \sum_{(i, j) \in \mathcal{A}} n^{s} t_{i j} x_{i j}^{s}
$$


subject to

$$
\begin{gathered}
\text { (H) } \min _{x \in\{0,1\}} \sum_{s \in \mathcal{S}} \sum_{(i, j) \in \mathcal{A}} n^{s}\left(c_{i j}\left(\check{v}_{i j}\right)+\sigma_{H} t_{i j}\right) x_{i j}^{s} \\
\sum_{(i, j) \in \mathcal{A}} x_{i j}^{s}-\sum_{(j, i) \in A} x_{j i}^{s}=e_{i}^{s} \quad \forall i \in N \quad \forall s \in S .
\end{gathered}
$$

We note that upon replacing problem $(\mathrm{H})$ by its primal-dual optimality conditions and linearizing the bilinear terms as described in Gupte et al. (2013), problem (HTS) is transformed into a single-level linear model which can be solved with a powerful linear programming software.

- For each arc $(i, j)$, evaluate $z_{i j}\left(L^{0}\right)$ and $z_{i j}\left(U^{0}\right)$. Set $k=1$ and go to step $k$.

\section{Step $k$. Iterative search for $\tau$}

- For each $(i, j)$, specify a new point $\tau_{i j}^{k} \in\left[L_{i j}^{k-1}, U_{i j}^{k-1}\right]$ using a line search procedure.

- Solve the UE problem (R) for given $\tau^{k}$ and get $v\left(\tau^{k}\right)$.

- Solve problem (HTS) for given $v\left(\tau^{k}\right)$ and obtain $\left.x(t)\right|_{\tau^{k}}$ and $\left.t\right|_{\tau^{k}}$.

- For each arc $(i, j)$, evaluate $z_{i j}\left(\tau^{k}\right)$.

- Calculate new intervals $\left[L_{i j}^{k}, U_{i j}^{k}\right]$, for each arc $(i, j)$ by a line search such as Golden Section.

- For all $(i, j)$, if $U_{i j}^{k}-L_{i j}^{k}<\Delta$, stop and set $\tau_{i j}^{*}=\left(L_{i j}^{k}+U_{i j}^{k}\right) / 2$; otherwise set $k=k+1$.

Since the line search procedure is based on function evaluations, the quality of the solution provided by the Modified EDO is very sensitive to the magnitudes of the risk and revenue terms. In particular, our numerical observations on the test problems of Table 2 reveal that for some cases, in the first iterations, large values of regular toll yield revenue terms of higher magnitudes. Hence, initially the algorithm favors the revenue term without making any significant improvement to the risk. Upon obtaining a revenue term with the same order of magnitude as risk, the algorithm starts minimizing both terms almost equally. At this stage, the intervals of $\tau$ are no longer wide enough to substantially minimize the risk, and consequently we can get low quality solutions. This motivates us to suggest a 2-Step EDO which resolves the shortcomings of the Modified EDO.

The 2-Step EDO uses the Modified EDO algorithm in two consecutive steps. Ignoring the revenue terms in problem (GTS), the first step applies the Modified EDO to obtain a solution $\left(\tau^{I}, v^{I}, t^{I}, x^{I}\right)$ which only minimizes the risk. From the underlying information in problem (GTS), one can notice that for any link $(i, j)$, regardless of the amount of regular flow (i.e., $v_{i j}^{I}$ ), without having a hazmat truck passing through the link, the corresponding risk value is zero implying that charging toll not only is neutral to risk reduction but also increases the revenue. Therefore, we incorporate the following modification to the regular toll vector, $\tau^{I}$, of the current solution:

$$
-\forall s \in \mathcal{S}, \forall(i, j) \in \mathcal{A}: \quad \text { if } x_{i j}^{s I}=0, \text { set } \tau_{i j}^{I}=0 ;
$$




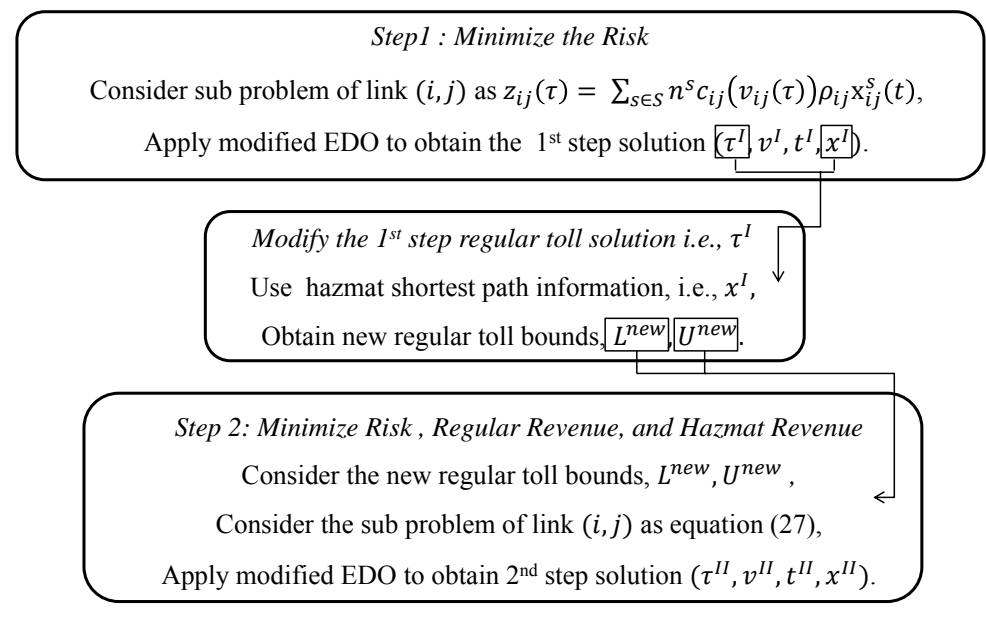

Figure 3: The 2-Step EDO algorithm

- Otherwise, $\quad$ set $L_{i j}^{\text {new }}=L_{i j}^{0}$ and $U_{i j}^{\text {new }}=\tau_{i j}^{I}$.

Starting from the new toll bounds, $L^{\text {new }}$ and $U^{\text {new }}$, the second step applies the Modified EDO to problem (GTS) to minimize risk and revenue simultaneously. In fact, modifying the first step solution helps the second step search procedure to start from the minimum risk solution and gradually redirect the exploration towards minimizing the revenue at the expense of a higher risk. This allows the algorithm to efficiently search in the direction of risk and revenue and lead to a qualified solution which favors both objective terms. Figure 3 illustrates the procedure undertaken by the 2-Step EDO algorithm.

In problem (GTS), one notable point is the possibility of obtaining multiple shortest path solutions to problem $(\mathrm{H})$ referred to as instability issue (Erkut and Gzara, 2008). Under such situations, the regulator's ability in minimizing the risk to its prescribed level is not guaranteed. Despite being theoretically possible, in real applications, multiple solutions to problem $(\mathrm{H})$ are very unlikely to happen due to numerical values generated by the BPR delay function during computations. Therefore, as problem (GTS) is supposed to address practical problems, we assume that a solution to problem $(\mathrm{H})$ is always stable.

To evaluate the performance of the Modified EDO and 2-Step EDO heuristics, we use the LINGO optimization solver as an exact method for problem (GTS). The details of our numerical analysis are found in the supplementary document (Appendix B). The results demonstrate that the CPU time substantially decreases under either of the heuristics, with the Modified EDO algorithm being in general superior to the 2-Step EDO. The 2-Step EDO is markedly more effective than the Modified EDO algorithm in terms of solution quality, providing nearly identical optimal solutions for all problem instances globally solved by LINGO. Also, for networks with significant congestion and complexity which cannot be handled by LINGO, the 2-Step EDO appears to be promising as a useful algorithm that can obtain quite good approximate solutions in significantly less amount of time. Therefore, for finding a locally optimal solution to problem (GTS), we suggest using the 2-Step EDO approach. 


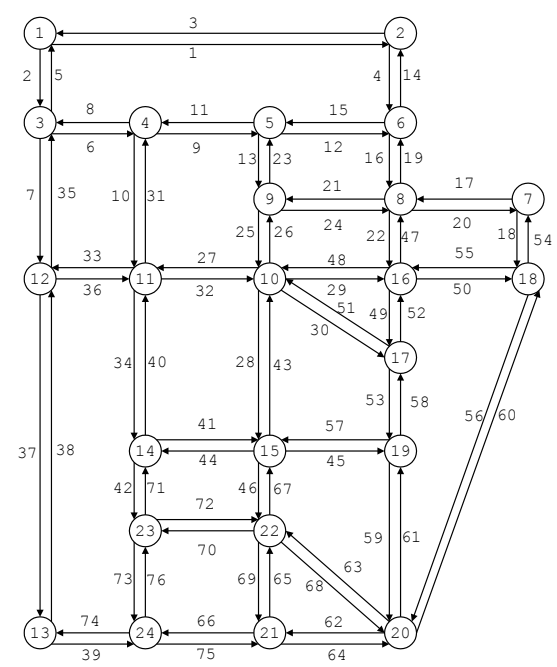

Figure 4: Sioux Falls Network

Furthermore, we notice that although, in majority of the test problems, the most risky arcs are subject to hazmat tolls, no tolls are paid by the carriers. In fact, one may misinterpret that hazmat tolls only serve to prohibit the carriers from using the corresponding arcs similar to the road ban policy. Thus, to avoid ambiguity, we provide an illustrative example in Appendix B. We show that the dual-toll policy has the advantage of not completely restricting the use of road segments and yet efficiently directing the drivers to less risky arcs based on their own considerations.

\section{Case Study on Sioux Falls Network}

As a basis for our case study, we use a network based on the roads of the city of Sioux Falls, South Dakota, consisting of 24 nodes and 76 arcs, as shown in Figure 4. We use the same network parameters of Suwansirikul et al. (1987, Table X) as the input parameters of the travel delay function $c_{i j}\left(v_{i j}\right)$. We also consider the original 552 OD pairs for the regular vehicles with the corresponding travel demands as displayed in Suwansirikul et al. (1987, Table XI). The Census site information provides an effective framework for estimating each arc $(i, j)$ population. Since there is no reliable record of the actual OD pairs used by the hazmat carriers in the region, hazmat shipments including origin, destination and demand are randomly generated assuming that all trucks contain the same hazmat type and pose the same level of exposure to the network.

\subsection{The Two-Stage Process}

We solved problem (TS) by the two-stage process depicted in Figure 2. To estimate parameters $\sigma_{R}$ and $\sigma_{H}$, a reasonable assumption is to consider the inverse of the Value of Time (VoT) of regular drivers and hazmat carriers, respectively. Various measures of VoT can be found in the literature each incorporating different travel cost factors. In particular, Litman (2009) estimates the travel 
time cost of a regular vehicle as $\$ 20.4 / \mathrm{hr}$ incorporating fuel cost, maintenance cost and insurance cost whereas Park et al. (2014) consider the operational cost of a truck to be $\$ 24.44 / \mathrm{hr}$ assuming the similar travel cost factors. According to this, the parameters $\sigma_{R}$ and $\sigma_{H}$ were set to 0.05 and 0.04 , respectively.

In order to better analyze the performance of the dual-toll policy, we compute the population exposure (risk), regular total travel delay and hazmat total travel delay for the unregulated network where no toll is charged to regular vehicles and hazmat trucks, i.e., no-toll case. For doing so, we identify the untolled UE flow pattern of regular vehicles, $v^{\text {no-toll }}$, satisfying the following VI:

$$
\sum_{(i, j) \in \mathcal{A}} c_{i j}\left(v_{i j}^{\text {no-toll }}\right)\left(v_{i j}-v_{i j}^{\text {no-toll }}\right) \geq 0 \quad \forall v \in V
$$

as well as the untolled hazmat flow pattern, $x^{\text {no-toll }}$, by solving the shortest path problem as below:

$$
x^{\mathrm{no}-\mathrm{toll}}=\arg \min _{x \in X} \sum_{s \in \mathcal{S}} \sum_{(i, j) \in \mathcal{A}} n^{s} c_{i j}\left(v_{i j}^{\mathrm{no}-\text { toll }}\right) x_{i j}^{s}
$$

Consequently, under the no-toll case, the network performance can be characterized by the following measures:

$$
\begin{aligned}
z\left(v^{\text {no-toll }}, x^{\text {no-toll }}\right) & =\sum_{s \in \mathcal{S}} \sum_{(i, j) \in \mathcal{A}} n^{s} c_{i j}\left(v_{i j}^{\text {no-toll }}\right) \rho_{i j} x_{i j}^{s \text { no-toll }}, \\
D_{R}\left(v^{\text {no-toll }}\right) & =\sum_{(i, j) \in \mathcal{A}} c_{i j}\left(v_{i j}^{\text {no-toll }}\right) v_{i j}^{\text {no-toll }} \\
D_{H}\left(v^{\text {no-toll }}, x^{\text {no-toll }}\right) & =\sum_{s \in \mathcal{S}} \sum_{(i, j) \in \mathcal{A}} n^{s} c_{i j}\left(v_{i j}^{\text {no-toll }}\right) x_{i j}^{s \text { no-toll }},
\end{aligned}
$$

where $z, D_{R}$, and $D_{H}$ denote the risk, regular vehicles delay and hazmat trucks delay, respectively.

For the dual-tolled case, we let $\left(v^{*}, x^{*}\right)$ denote the minimum-risk flow solution obtained from the post-iteration step, and $\left(\tau^{*}, t^{*}\right)$ be the dual-toll policy determined by $\operatorname{RTP}\left(v^{*}\right)$ and $\operatorname{HTP}\left(x^{*}\right)$.

Equipped with the above definitions, to compare the dual-tolled network with its no-toll counterpart, we compute the $\%$ change in risk, regular travel delay and hazmat travel delay using the following metrics:

$$
\begin{aligned}
\% \text { Change of Risk } & =\frac{z\left(v^{*}, x^{*}\right)-z\left(v^{\text {no-toll }}, x^{\text {no-toll }}\right)}{z\left(v^{\text {no-toll }}, x^{\text {no-toll }}\right)} \times 100 \\
\% \text { Change of Delay (Regular }) & =\frac{D_{R}\left(v^{*}\right)-D_{R}\left(v^{\text {no-toll }}\right)}{D_{R}\left(v^{\text {no-toll }}\right)} \times 100 \\
\% \text { Change of Delay }(H a z m a t) & =\frac{D_{H}\left(v^{*}, x^{*}\right)-D_{H}\left(v^{\text {no-toll }}, x^{\text {no-toll }}\right)}{D_{H}\left(v^{\text {no-toll }}, x^{\text {no-toll }}\right)} \times 100
\end{aligned}
$$

For the dual-tolled problem, we also report the average amount of tolls collected from an individual, namely, $\frac{\sum_{(i, j) \in \mathcal{A}} \tau_{i j}^{*} v_{i j}^{*}}{\sum_{w \in \mathcal{W}} d^{w}}$ and $\frac{\sum_{s \in \mathcal{S}} \sum_{(i, j) \in \mathcal{A}} n^{s} t_{i j}^{*} x_{i j}^{s}{ }^{*}}{\sum_{s \in \mathcal{S}} n^{s}}$ for regular drivers and hazmat carriers, respectively. 
Table 4: Dual-toll performance when compared with no-toll case under different numbers of regular and hazmat OD pair with first-stage problem as (MR)

\begin{tabular}{|c|c|c|c|c|c|c|c|}
\hline \multirow[b]{2}{*}{ Case } & \multicolumn{2}{|c|}{ No. of OD pairs } & \multirow{2}{*}{$\begin{array}{r}\text { \% Change } \\
\text { of Risk }\end{array}$} & \multicolumn{2}{|c|}{$\%$ Change of Delay } & \multicolumn{2}{|c|}{ Avg. Tolls per Individual } \\
\hline & Regular & Hazmat & & Regular & Hazmat & Regular & Hazmat \\
\hline 1 & 10 & 2 & -25.35 & 304.43 & 16.63 & $0^{+}$ & 0 \\
\hline 2 & 10 & 5 & -15.07 & 84.33 & 10.81 & 0.96 & 0 \\
\hline 3 & 10 & 10 & -12.65 & 214.77 & 11.97 & 1.43 & 0 \\
\hline 4 & 20 & 10 & -12.64 & 170.32 & 11.96 & 0.36 & 0 \\
\hline 5 & 20 & 15 & -8.43 & 116.91 & 8.55 & 1.57 & 0 \\
\hline 6 & 20 & 20 & -6.23 & 94.04 & 11.35 & 1.95 & 0.12 \\
\hline 7 & 50 & 20 & -6.68 & 65.84 & 10.68 & 0.53 & 0.12 \\
\hline 8 & 100 & 20 & -7.27 & 207.81 & 8.28 & 4.50 & 0.12 \\
\hline 9 & 200 & 20 & -12.55 & 1679.94 & 2.61 & 174547.38 & 0.09 \\
\hline 10 & 400 & 20 & -41.60 & 31709.63 & -33.20 & 29383941.26 & 0.11 \\
\hline 11 & 552 & 10 & -63.36 & 18471.56 & -60.30 & 15526713.71 & 0 \\
\hline
\end{tabular}

The results of our numerical experiments under different numbers of regular and hazmat OD pairs are reported in Table 4. From Table 4, it is evident that the exposure to hazmat transportation can be significantly reduced under the dual-toll policy. However, this requires the regular vehicles to incur an increase in their travel time comparing to the no-toll scenario. Although one may predict a similar behavior for hazmat carriers' travel delay, from case 11 we notice that when all regular OD pairs are considered, i.e., the congested network case, a higher decrease in the risk can be achieved at the expense of substantial increase in regular travel delay, whereas the hazmat travel delay is reduced to less than the half of its value in the no-toll case.

It is important to note that the change of delay for hazmat carriers is affected by the network congestion. In particular, for the cases with lower congestion, we observed that the dual-toll policy detours the hazmat carriers into longer routes which are not being used by regular vehicles. Therefore, it leads to an increase in the carriers travel delay since the congestion induced by regular vehicles in the no-toll case is negligible. Cases with positive \% change of delay for hazmat carriers refer to such situation. On the other hand, when the network is highly congested, the dual-toll deviates hazmat carriers into less congested routes to mitigate the risk exposure, and consequently results in a decrease in their travel delay.

One can also see from Table 4 that the reduction of risk is often achieved by collecting tolls from the regular vehicles. Nevertheless, when the number of hazmat OD pairs is large enough (above 15 for the network considered), owing to the similar reasoning described in Appendix B for the illustrative example, such reduction is obtained by collecting tolls from both types of traffic.

So far, our results indicate that the dual-toll policy, motivating the regular and hazmat traffic to use divergent routes, is an effective tool for risk-mitigation which produces attractive solutions for the regulator. However, since the regulator does not directly incorporate the users' viewpoint in its decision-making process, some users may have to incur unbearable time and financial burdens 
Table 5: Dual-toll performance when compared with no-toll case under different numbers of regular and hazmat OD pair, with first-stage problem as equation (30) and the weight parameters as $\omega_{1}=0.5, \omega_{2}=0.5$, and $\omega_{3}=0$

\begin{tabular}{|c|c|c|c|c|c|c|c|}
\hline \multirow[b]{2}{*}{ Case } & \multicolumn{2}{|c|}{ No. of OD pairs } & \multirow{2}{*}{$\begin{array}{r}\% \text { Change } \\
\text { of Risk }\end{array}$} & \multicolumn{2}{|c|}{$\%$ Change of Delay } & \multicolumn{2}{|c|}{ Avg. Tolls per Individual } \\
\hline & Regular & Hazmat & & Regular & Hazmat & Regular & Hazmat \\
\hline 1 & 10 & 2 & -25.12 & 0 & 17.02 & 0 & 0 \\
\hline 2 & 10 & 5 & -14.95 & 0 & 10.99 & 0 & 0 \\
\hline 3 & 10 & 10 & -12.57 & 0 & 12.09 & 0 & 0 \\
\hline 4 & 20 & 10 & -12.53 & 0 & 12.14 & 0 & 0 \\
\hline 5 & 20 & 15 & -8.35 & 0 & 8.65 & 0 & 0 \\
\hline 6 & 20 & 20 & -6.17 & 0 & 11.44 & 0 & 0.12 \\
\hline 7 & 50 & 20 & -6.51 & -0.50 & 10.89 & 0.02 & 0.12 \\
\hline 8 & 100 & 20 & -6.62 & -0.69 & 10.88 & 0.18 & 0.11 \\
\hline 9 & 200 & 20 & -10.61 & -1.05 & 6.56 & 0.45 & 0.09 \\
\hline 10 & 400 & 20 & -27.10 & 0.03 & -19.11 & 1.20 & 0 \\
\hline 11 & 552 & 10 & -24.39 & -0.01 & -5.55 & 1.32 & 0 \\
\hline
\end{tabular}

under the dual-tolled network. Note the substantially increased travel delay as well as the average tolls paid by an individual regular vehicle in Table 4. Clearly, such policies would not be feasible to the users and the regulator has to ensure their satisfaction in the implementation phase. Hence, to obtain solutions that can be more acceptable to the network users, we suggest replacing equation (17) with a new administrator's problem of the following form:

$\min _{v \in V, x \in X} J(v, x)=\omega_{1} \sum_{s \in \mathcal{S}} \sum_{(i, j) \in \mathcal{A}} n^{s} c_{i j}\left(v_{i j}\right) \rho_{i j} x_{i j}^{s}+\omega_{2} \sum_{(i, j) \in \mathcal{A}} c_{i j}\left(v_{i j}\right) v_{i j}+\omega_{3} \sum_{s \in \mathcal{S}} \sum_{(i, j) \in \mathcal{A}} n^{s} c_{i j}\left(v_{i j}\right) x_{i j}^{s}(30)$

with problems $(R)$ and $(H)$ being the same as in Section 3.1 in the second-stage, and $\omega_{1}, \omega_{2}, \omega_{3} \in[0,1]$, $\sum_{i} \omega_{i}=1$, the weight parameters, addressing the relative significance of each term.

As one can note, other than the risk exposure term, in equation (30), we incorporate the regular total travel delay, network congestion, and the hazmat total travel delay, hoping to obtain implementable solutions wherein the administrator compromises its risk mitigation targets for the sake of the users' travel time.

To evaluate the efficiency of the provided dual-toll under the new administrator's problem, we repeated all the experiments of Table 4, with equation (17) being replaced with (30) in the two-stage process of Figure 2. Note that due to having similar structural properties as (MR), an approximate solution to equation (30) can still be found by the Modified Frank-Wolfe algorithm. Table 5 presents the results of our computational tests based on the aforementioned metrics.

An interesting observation, when comparing each problem instance of Table 5 with its counterpart in Table 4, is that a slightly less reduction of risk is achieved with no increase in regular travel delay and slightly higher increase in hazmat travel delay. In fact, with a few number of regular OD pairs considered, the congestion is very low leading to no change in the UE flow pattern and no tolls 
being collected from regular vehicles. However, now hazmat carriers have to incur higher travel times to avoid risky arcs. For cases with higher congestion, i.e., starting from case 7, the dual-toll policy has the advantage of directing the regular vehicles to the less risky arcs while reducing their travel delay at the expense of viable tolls paid by the drivers. Similar argument to low congestion cases applies for hazmat carriers travel delay. Nonetheless, when the network is highly congested, i.e., cases 10 and 11, hazmat travel delay also decreases since hazmat carriers are directed into less congested areas compared with the no-toll case.

It is important to notice that for all problem instances of Table 5 we consider $\omega_{3}=0$ due to the assumption that congestion induced by hazmat carriers is negligible. Consequently, the dual-tolled transportation network is in favor of regular vehicles. In a real application, being aware of the potential risk imposed by hazmat trucks to the network, it might be acceptable to the corresponding transportation companies to use more expensive routes. However, more attractive solutions for hazmat carriers can be obtained by considering a nonzero $\omega_{3} \in(0,1]$, at the cost of deteriorating risk mitigation targets and regular vehicles' viewpoint.

In summary, our numerical experiments have shown that with the inclusion of the network users' travel delay within the first-stage problem, i.e., equation (30), other than congestion minimization, the risk is significantly reduced and is only slightly higher than that obtained under equation (17). Therefore, the two-stage process with the new administrator's problem becomes more interesting for implementation because it produces far less expensive solutions for the network users (regular vehicles in our case) which are yet attractive to the regulator.

We also experiment the case where tolls are only charged to hazmat carriers, i.e., single-toll case, to further evaluate the impact of the dual-toll policy with respect to the single-toll policy. Table 6 presents our results under two different scenarios of the leader's objective for cases 9,10 and 11 of Tables 4 and 5. We utilize a two-stage procedure analogous to the one introduced by Marcotte et al. (2009) to solve the single-toll problem, and compute the percentage changes in population exposure (risk), hazmat total travel delay, and the average toll collected from each hazmat carrier from single-toll to dual-toll case. Note that for regular vehicles the percentage change of the total travel delay and the average tolls collected are identical to the ones illustrated in Tables 4 and 5, thereby such results are not reported again.

As one can predict the single-toll policy can reduce the hazmat transport risk at the cost of tolls paid by the carriers. Whereas the regular vehicles follow the UE flow pattern without paying tolls. From Table 6, it is evident that, when the regulator's objective is solely to minimize the population exposure, the dual-toll policy can achieve significantly higher reductions in the associated transport risk, hazmat travel delay as well as the tolls paid by the carriers comparing to the single-toll policy. The regular vehicles, however, experience unbearable increases in their travel time and the paid tolls. When the administrator incorporates the regular travel delay in its decision making process, the dual-toll still produces more attractive solutions than the single-toll policy in terms of risk, travel delay and the paid tolls incurred by hazmat carriers. Furthermore, this involves no increase in regular travel delay at the expense of feasible tolls paid by the drivers. 
Table 6: Dual-toll performance with respect to the single-toll policy under two considerations of the first-stage problem and different numbers of regular and hazmat OD pair

\begin{tabular}{|c|c|c|c|c|c|}
\hline \multirow{2}{*}{$\begin{array}{l}\text { First-Stage } \\
\text { Problem }\end{array}$} & \multicolumn{2}{|c|}{ No. of OD pairs } & \multirow{2}{*}{$\begin{array}{r}\text { \% Change } \\
\text { of Risk }\end{array}$} & \multirow{2}{*}{$\begin{array}{r}\% \text { Change of Delay } \\
\text { for Hazmat }\end{array}$} & \multirow{2}{*}{$\begin{array}{l}\text { \% Change in Avg. Tolls } \\
\text { Collected from Hazmat }\end{array}$} \\
\hline & Regular & Hazmat & & & \\
\hline \multirow{3}{*}{ Eq. $(17)^{a}$} & 200 & 20 & -5.80 & -7.09 & -1.35 \\
\hline & 400 & 20 & -33.21 & -39.98 & -32.29 \\
\hline & 552 & 10 & -57.75 & -63.55 & 0 \\
\hline \multirow{3}{*}{ Eq. $(30)^{b}$} & 200 & 20 & -3.70 & -3.51 & 0.78 \\
\hline & 400 & 20 & -16.62 & -27.32 & -100 \\
\hline & 552 & 10 & 12.80 & -13.26 & 0 \\
\hline
\end{tabular}

\footnotetext{
${ }^{a}$ Refers to the case where the administrator's only concern is the risk exposure, i.e., problem (MR)

${ }^{b}$ Refers to the case where the administrator's concern is not only the risk exposure but also the regular total travel delay and the hazmat total travel delay. The weight parameters are set to $\omega_{1}=0.5, \omega_{2}=0.5$, and $\omega_{3}=0$.
}

Table 7: Performance of the dual-toll policy provided by the 2-step EDO algorithm versus the no-toll case

\begin{tabular}{ccccc}
\hline $\begin{array}{c}\text { Change } \\
\text { of Risk }(\%)\end{array}$ & $\begin{array}{c}\text { Change of Delay } \\
(\text { Regular })(\%)\end{array}$ & $\begin{array}{c}\text { Change of Delay } \\
(\text { Hazmat })(\%)\end{array}$ & \multicolumn{2}{c}{ Avg. Tolls per Individual } \\
\cline { 4 - 5 } & 23.57 & -14.33 & 0.19 & 0 \\
\hline
\end{tabular}

\subsection{The 2-Step EDO}

We solve problem (GTS) using the 2-Step EDO heuristic depicted in Figure 3 as a true bilevel model. For our testing, all 552 OD pairs as displayed in Suwansirikul et al. (1987, Table XI) were considered for the regular vehicles, whereas 10 hazmat trips including origin, destination and demand were generated randomly. Also, we assumed that 18 arcs are subject to charge by separate tolls for regular and hazmat users with 20 and 30 being the upper bounds on regular and hazmat tolls, respectively. Tollable link numbers in Figure 4 are: 7, 10, 13, 16, 19, 23, 27, 31, 32, 35, 41, 44, 62, $64,65,69,70$, and 72. Also, we let $\sigma_{R}=0.05, \sigma_{H}=0.04$ as in the previous section, and $\varphi=0.1$. With these assumptions, the 2-Step EDO heuristic was employed to obtain a dual-toll policy. The results are presented in Table 7 according to the similar metrics defined in Section 6.1.

It is important to note that due to the inclusion of a fraction of the carriers' cost within the leader's objective of problem (GTS), the reduction in risk is not as high as the one produced by problem (TS). Note the difference between the results of Table 7 and the ones in case 11 of Table 4 . Further, when some road segments are free of toll charges, i.e., second-best pricing, the optimal value of the risk objective function is likely to deteriorate. Nonetheless, it is evident from Table 7 that the exposure to hazmat transportation can be still substantially reduced by the dual-toll policy obtained from problem (GTS). Also, for such reduction, the regular vehicles incur far less increase in their travel delay and paid tolls. Hazmat carriers, on the other hand, are directed into less congested routes and thus experience shorter travel times without paying tolls. 
For our test problem, we also obtained the single-toll policy by solving the single-level MIP reformulation. We observed that although the dual-toll policy involves similar impacts on the regular travel delay and the paid tolls as in Table 7, the reductions in the risk and the hazmat travel time under such policy are respectively $16 \%$ and 19\% higher than those obtained from single-toll policy.

In summary, the dual-toll obtained from the 2-Step EDO heuristic, can effectively encourage regular vehicles and hazmat carriers to use dissimilar routes, and thus can achieve considerable reductions in the associated transport risk and the travel delay experienced by hazmat carriers while collecting much less tolls from the users. The results imply that, by combining the risk and the paid tolls, problem (GTS) can yield attractive solutions for the regulator which are also economically feasible to the users. Although, it might be perceived as less fair from regular users in terms of the travel delay, when the leader's objective of problem (GTS) also allows for minimizing the congestion induced by regular vehicles, the 2-Step EDO heuristic can produce more acceptable solutions to the regular vehicles at the cost of compromising the risk and the paid tolls.

\section{Conclusion and Future Research}

This paper has considered dual-toll setting, with a nonlinear BPR travel delay function, as an efficient policy for mitigating the population exposure to dangerous goods transportation. We demonstrated that the bilevel problem (TS), first-best pricing, is equivalent to a two-stage problem with (MR) being in the first-stage and regular/hazmat inverse optimizations in the second-stage. It is important to stress that, under the nonlinear BPR function, a numerical solution to problem (MR) may be sub-optimal; thus the existence of a nonnegative valid toll vector pair may not be guaranteed. Based upon analytical properties, this paper has proposed a post-iteration to the numerical solution to (MR) which always ensures obtaining a nonnegative dual-toll policy inducing the minimum hazmat risk. We suggested using the Modified Frank-Wolfe heuristic to determine an approximate solution to problem (MR). Further, to study the quality of this solution, we transformed the nonlinear problem (MR) to two MILPs that assure global optimality and serve to justify the approximate solution obtained by the heuristic. Numerical results show that the heuristic algorithm converges to the optimal solution of the administrator's problem (MR) with reduced computational effort.

We also studied a general dual-toll setting problem, namely (GTS), where the regulator wishes to minimize a combination of hazmat transport risk and paid tolls in a framework that permits constraints on toll charges, second-best pricing. A 2-Step EDO heuristic is proposed to solve (GTS), by decomposing it into interacting univariate subproblems for each arc and applying simultaneous line searches. The (GTS) problem was also solved using LINGO as an exact method to verify heuristic results. Our experiments revealed that the 2-Step EDO is computationally more efficient than LINGO and converges to the local optimal solution of (GTS).

To illustrate the effectiveness of the dual-toll policies provided by problems (TS) and (GTS), we carried out experiments with the Sioux Falls problem. We observed that, under the dual-toll 
obtained from (TS), the exposure to hazmat transportation can be significantly reduced, yielding attractive scenarios for the regulator. Nevertheless, our numerical tests showed that such scenarios are substantially expensive from the users' perspective in terms of both the travel delay and the paid tolls; thus they would likely not be acceptable by users in the implementation phase. To overcome this, we suggest incorporation of a fraction of the users' travel delay within the administrator's objective function. We demonstrated that the new (TS), while compromising risk-mitigation targets to minimize the travel delay, is still able to achieve significant reductions in risk, and can produce win-win scenarios for both the regulator and the network users. Another observation relates to problem (GTS). Although combining the risk and the paid tolls most likely deteriorates the risk reduction when compared with (TS), we show that the solutions obtained under such dual-toll are not only likely to be attractive for the regulator but also likely to be financially acceptable to users.

One limitation of our work is the impossibility of including the regular traffic flow rate in the number of people exposed to a hazmat accident due to incompatible units. Although our experiments showed that, with the current risk measurement, hazmat carriers are implicitly avoiding highly congested areas due to incorporation of the travel delay function, a valid future extension would be to also account for the regular vehicles exposure in the risk assessment model.

A challenging problem in hazmat transportation is equity in distributing the risk among the different population centers. Hence, a suggested future refinement of our model is to obtain a dual-toll setting policy which also includes equity concerns of the public. Another important issue pertinent to network regulation is the inevitable increase in the users' travel cost. Although the dual-toll setting model, by incorporating the network users' cost concerns, can substantially reduce the overall travel cost imposed to the users, such benefit is not equitably distributed. In fact, a detailed analysis of the solution shows that, while being more fair to some users, some individuals have to sacrifice for the sake of total system satisfaction. Therefore, another fruitful direction is to integrate cost equity among the network users in the decision-making process.

\section{Acknowledgment}

This manuscript is based upon work supported by the National Science Foundation under Grant Number CMMI-1068585. Any opinions, findings, and conclusions or recommendations expressed in this manuscript are those of the authors and do not necessarily reflect the views of the National Science Foundation. This research was done while the first and second authors were in the Department of Industrial and Systems Engineering at the University at Buffalo. 


\section{References}

Abkowitz, M., P. D.-M. Cheng. 1988. Developing a risk/cost framework for routing truck movements of hazardous materials. Accident Analysis \& Prevention 20(1) 39-51.

Ahuja, R. K., J. B. Orlin. 2001. Inverse optimization. Operations Research 49(5) 771-783.

Arnott, R., K. Small. 1994. The economics of traffic congestion. American Scientist 446-455.

Batta, R., S. S. Chiu. 1988. Optimal obnoxious paths on a network: Transportation of hazardous materials. Operations Research 36(1) 84-92.

Bergendorff, P., D. W. Hearn, M. V. Ramana. 1997. Congestion toll pricing of traffic networks. Network Optimization 450 51-71.

Bianco, L., M. Caramia, S. Giordani. 2009. A bilevel flow model for hazmat transportation network design. Transportation Research Part C: Emerging Technologies 17(2) 175-196.

Bianco, L., M. Caramia, S. Giordani, V. Piccialli. 2015. A game-theoretic approach for regulating hazmat transportation. Transportation Science Articles in Advance. URL http://dx.doi. org/10.1287/trsc.2015.0592.

Dafermos, S. 1980. Traffic equilibrium and variational inequalities. Transportation Science 14(1) $42-54$.

Erkut, E., O. Alp. 2007. Designing a road network for hazardous materials shipments. Computers \& Operations Research 34(5) 1389-1405.

Erkut, E., F. Gzara. 2008. Solving the hazmat transport network design problem. Computers $\&$ Operations Research 35(7) 2234-2247.

Florian, M., D. Hearn. 1995. Network equilibrium models and algorithms. Handbooks in Operations Research and Management Science 8 485-550.

Frank, M., P. Wolfe. 1956. An algorithm for quadratic programming. Naval Research Logistics Quarterly 3(1-2) 95-110.

Fukushima, M. 1984. A modified frank-wolfe algorithm for solving the traffic assignment problem. Transportation Research Part B: Methodological 18(2) 169-177.

Gupte, A., S. Ahmed, M. S. Cheon, S. Dey. 2013. Solving mixed integer bilinear problems using MILP formulations. SIAM Journal on Optimization 23(2) 721-744.

Hearn, D. W., M. V. Ramana. 1998. Solving congestion toll pricing models. Springer.

Johansson-Stenman, O., T. Sterner. 1998. What is the scope for environmental road pricing?. Road Pricing, Traffic Congestion and the Environment: Issues of Efficiency and Social Feasibility . 
Kang, Y., R. Batta, C. Kwon. 2011. Value-at-risk model for hazardous material transportation. Annals of Operations Research 1-27.

Kara, B. Y., V. Verter. 2004. Designing a road network for hazardous materials transportation. Transportation Science 38(2) 188-196.

Litman, T. 2009. Transportation cost and benefit analysis. Victoria Transport Policy Institute 1-19.

Marcotte, P., A. Mercier, G. Savard, V. Verter. 2009. Toll policies for mitigating hazardous materials transport risk. Transportation Science 43(2) 228-243.

Park, J., C. Kwon, M. Son. 2014. Economic implications of the canada-us border bridges: Applying a binational local economic model for international freight movements. Research in Transportation Business \& Management 11 123-133.

Suwansirikul, C., T. L. Friesz, R. L. Tobin. 1987. Equilibrium decomposed optimization: a heuristic for the continuous equilibrium network design problem. Transportation Science 21(4) 254-263.

Toumazis, I., C. Kwon, R. Batta. 2013. Value-at-risk and conditional value-at-risk minimization for hazardous materials routing. Handbook of OR/MS Models in Hazardous Materials Transportation. Springer, 127-154.

Wang, D. Z., H. K. Lo. 2010. Global optimum of the linearized network design problem with equilibrium flows. Transportation Research Part B: Methodological 44(4) 482-492.

Wang, J., Y. Kang, C. Kwon, R. Batta. 2012. Dual toll pricing for hazardous materials transport with linear delay. Networks and Spatial Economics 12(1) 147-165.

Yang, H., H.-J. Huang. 2004. The multi-class, multi-criteria traffic network equilibrium and systems optimum problem. Transportation Research Part B: Methodological 38(1) 1-15.

Yin, Y., S. Lawphongpanich. 2006. Internalizing emission externality on road networks. Transportation Research Part D: Transport and Environment 11(4) 292-301. 